\title{
Etnopedologia: uma abordagem das etnociências sobre as relações entre as sociedades e os solos
}

\author{
Ethnopedology: an ethnosciences approach on relations between societies and soils
}

\author{
Ana Leônia de Araújo $^{\mathrm{I}^{*}}$ Ângelo Giuseppe Chaves Alves ${ }^{\mathrm{II}}$ Ricardo Espíndola Romero ${ }^{\mathrm{I}}$ \\ Tiago Osório Ferreira ${ }^{\mathrm{I}}$
}

\section{- REVISÃO BIBLIOGRÁFICA -}

\section{RESUMO}

A avaliação do conjunto de conhecimentos gerados e transmitidos por populações humanas caracteriza-se como o campo de atuação dos estudos etnocientíficos. Esta avaliação de conhecimentos relacionados aos solos e sua associação com recursos naturais, bem como estratégias desenvolvidas para produção são os objetos de estudo da etnopedologia. A utilização de metodologias participativas que promovam a inserção direta dos envolvidos na pesquisa são premissas básicas nos trabalhos etnopedológicos. As áreas de avaliação etnopedológicas relacionam-se, dentre outros aspectos, a nomenclaturas para classificação das terras e práticas de manejo. $O$ crescente desenvolvimento etnopedológico revela o reconhecimento da importância do solo como um recurso limitado e essencial para a vida e reforça uma visão conservacionista importante a ser considerada na construção de trabalhos na área de ciências do solo.

Palavras-chave: metodologias participativas, conhecimento, agricultores.

\section{ABSTRACT}

The overall assessment of knowledge produced and transmitted by a human group is set as ethnoscience field. This evaluation of information related to soils and its relation with natural resources, as well as strategies developed to production, are the scope of study from ethnopedology. Participatory methodologies which promote the inclusion of those directly involved are basic assumptions of ethnopedology. Areas of evaluation from ethnopedology studies are related, among other aspects, to nomenclatures for soil classification and management practices. The increasing ethnopedological development reveals soil importance recognition as a limited and essential resource for life and strengthens an important conservationist view to be considered in researches of soil science.

Key words: participatory methodologies, knowledge, farmers.

\section{INTRODUÇÃO}

As etnociências baseiam-se em avaliações antropológicas que consideram o saber como um conjunto de habilidades possíveis de serem transmitidas e objetivam avaliar os princípios e pressupostos desse conhecimento, considerando a possibilidade de estabelecer relações com o conhecimento científico formal (ALVES \& MARQUES, 2005).

O conhecimento das populações estudadas pode ser referenciado de diferentes formas pelas etnociências, tais como: conhecimento local, indígena, popular, nativo, tradicional, entre outros (ALVES \& MARQUES, 2005). Neste trabalho, emprega-se o termo "formal" para referir-se ao conhecimento do pesquisador e o termo "local" para o das populações em estudo. AGRAWAL (1995) salienta, no entanto, que a distinção entre as duas formas de conhecimento não é o mais importante, mas sim a que lógicas elas se referem, já que ambas possuem características comuns, ao mesmo tempo em que apresentam diferenças internas.

O termo etnoecologia tem sido usado para se referir aos estudos que descrevem as interações das populações humanas com todos os aspectos do ambiente natural, incluindo plantas, animais, paisagens, florestas e solos, entre muitos outros temas (MARTIN, 1995). Dessa forma, a etnoecologia

'Departamento de Ciências do Solo, Universidade Federal do Ceará (UFC), Campus do Pici, Av. Mister Hull, 2977, Bloco 807, Pici, 60021-970, Fortaleza, CE, Brasil. E-mail: analeonia@yahoo.com.br. *Autor para correspondência.

"Departamento de Biologia, Universidade Federal Rural de Pernambuco (UFRPE), Recife, PE, Brasil. 
reúne em si outros enfoques mais específicos como etnopedologia, etnobotânica e etnoastronomia, por exemplo. Também, para TOLEDO (2000), a etnopedologia é uma parte da etnoecologia.

Considerando as ciências com abordagem tradicionalmente técnica, a ciência do solo teve em seus anos iniciais uma atuação ligada especificamente à produção de alimentos, fibras e combustíveis. No entanto, nas três últimas décadas, essa área de estudo também direcionou ações para incluir aspectos sociais, ambientais e ecológicos em seus objetos de estudo, (WILDING \& LIN, 2006) percebendo que pesquisas sobre a importância dos solos precisavam ser abordadas de forma mais integrada, mas mantendo o rigor científico para que as ideias não fossem refutadas por tomadores de decisão (HARTEMINK \& MCBRATNEY, 2008). Aliados a essa tendência, vários pesquisadores têm buscado novas formas de atuar, envolvendo públicos diversos do meio científico para trabalhar conjuntamente as formas de compreensão sobre os solos (BRIDGES et al., 1996; MUGGLER et al., 2006; MEGONIGAL et al., 2010). Utilizando-se metodologias e experiências com abordagens envolventes que valorizam o conhecimento prévio, tem-se incrementado a atuação nesse promissor campo de pesquisa. Essas ações estão associadas com abordagens etnopedológicas, já que estas propõem o diálogo entre pesquisadores e agricultores de forma a construir um saber mais integrado e aplicado, objetivando a ampliação do reconhecimento do solo como um recurso natural.

Avaliação dos contextos cultural, social, econômico e ambiental, divulgação dos solos e integração de conhecimentos são tópicos que relacionam a ciência do solo e a etnopedologia. Sendo assim, objetivou-se destacar a importância da etnopedologia como uma abordagem integradora de conhecimentos, demonstrada através de produções científicas, dando-se ênfase nas experiências metodológicas e sua avaliação, bem como contribuições para o aprimoramento da relação entre conhecimento formal e local.

A etnopedologia e sua importância

A etnopedologia foi definida como uma disciplina híbrida estruturada na combinação das ciências naturais e sociais (BARRERA-BASSOLS \& ZINCK, 2000) com foco nas teorias locais sobre a dinâmica do solo e suas propriedades, envolvendo o manejo e percepção das relações solo-planta (WILLIAMS \& ORTIZ- SOLORIO, 1981). Pode-se caracterizá-la ainda, como um meio para compreender a realidade local e a relação com o recurso solo de um determinado grupo de pessoas.
O conhecimento local sobre solos baseia-se na experimentação, possui raízes onde se vive, é transmitido oralmente ou pela prática, está sensivelmente relacionado com as condições socioculturais e climáticas onde se insere e tem caráter dinâmico (BARRIOS \& TREJO, 2003; PAYTON et al., 2003). A forma como as pessoas observam e mensuram os recursos ao seu redor, como solucionam problemas e validam novas informações, também são considerados como componentes do conhecimento local (FAO, 2004).

A crescente preocupação científica com desenvolvimento sustentável e uso adequado dos recursos naturais fez com que pesquisadores e extensionistas, da área da Agronomia e afins, se aproximassem da etnopedologia, desenvolvendo estudos com abordagens ligadas à correlação e comparação entre o conhecimento formal e local, especialmente sobre aspectos relacionados à classificação de solos e estratégias de adaptação para o desenvolvimento da agricultura (NIEMEIJER \& MAZZUCATO, 2003).

Essa aproximação pode ser observada no trabalho de BARRERA-BASSOLS \& ZINCK (2003) que, avaliando a situação das publicações sobre etnopedologia no contexto mundial, reuniram 895 referências, das quais $48 \%$ possuíam a etnopedologia como objetivo central, focando especificamente na análise da percepção, conhecimento e manejo do solo. Os 52\% restantes englobaram trabalhos com abordagem que forneciam dados etnopedológicos dentro de um contexto de pesquisa mais amplo. Os dados confirmaram que de 1989 a 1998 houve um considerável aumento nas pesquisas etnopedológicas, com uma média de produção de 33 trabalhos por ano. A distribuição desses trabalhos também foi avaliada, sendo que $35 \%$ concentram-se na África, 34\% na América, 26\% na Ásia, 4\% na Europa e 1\% em ilhas localizadas no Oceano Pacífico. O país com maior número de trabalhos foi o México, com 70 trabalhos referenciados, enquanto o Brasil figurou naquele levantamento com 13 trabalhos.

Além da importância reconhecida pelo aumento do número de trabalhos nessa área, destacase que estudos etnopedológicos proporcionam a compreensão da realidade local de uma população humana e geram conhecimentos de forma mais acessível e integrada com os agricultores (CORREIA, 2005). Dessa forma, o desenvolvimento de tecnologias e os trabalhos de extensão rural podem ter maior transcendência e aplicabilidade através de uma abordagem etnopedológica (BIRMINGHAM, 2003). 
Avaliando a estruturação do conhecimento local, TOLEDO (2000) sugere que esta é formada por três domínios: kosmos-corpus-praxis. O kosmos refere-se aos sistemas de crenças e troca de simbolismos. O domínio do corpus refere-se ao sistema cognitivo, como o conjunto de conhecimentos sobre uso e manejo dos recursos naturais, enquanto a praxis está ligada ao conjunto de práticas desenvolvidas por um determinado grupo de pessoas.

Metodologias e análise de dados em etnopedologia

Diversas experiências metodológicas têm sido documentadas em estudos etnopedológicos (BARRERA-BASSOLS \& ZINCK, 2003). Salientase que as documentações referem-se, principalmente, a metodologias participativas ou integradoras. A escolha de metodologias com essa abordagem reside no fato de elas prezarem por uma relação mais próxima entre pesquisado e pesquisador, podendo contar com a participação de pesquisadores de várias áreas de estudo, numa perspectiva de ampliar a capacidade de compreensão das diferentes formas de manifestação das informações e complementaridade dos conhecimentos entre as perspectivas formal e local.

As formas usadas para a aquisição do conhecimento local são definidoras da qualidade das informações. A combinação de diferentes estratégias pode permitir a escolha de formas mais adequadas, que podem variar em cada situação estudada (OUDWATER \& MARTIN, 2003). O uso de entrevistas é considerado uma forma acessível de obtenção de informações. CORREIA (2005) e ALVES (2004) optaram pela utilização de entrevistas semi-estruturadas, que consistem na definição de um tema, a partir do qual se constrói uma série de informações relacionadas, de acordo com o entrosamento do entrevistado.

A utilização de cadernetas de campo para anotação de informações ou resumos diários dos trabalhos desenvolvidos é importante como fonte de documentação, além do uso de gravadores de voz e fotografias. É importante ressaltar que, por uma questão de ética, é imprescindível solicitar a concordância ou anuência prévia dos entrevistados, através do Termo de Consentimento Livre e Esclarecido, de acordo com a resolução no 292 , de 08/07/1999, do Conselho Nacional de Saúde (BRASIL, 1999).

$\mathrm{Na}$ busca por ações para incrementar a avaliação de agroecossistemas e de elementos para elucidar como acontece a relação destes com os grupos sociais, DUFUMIER (2007) propõe a metodologia intitulada "Diagnóstico de sistemas agrários", que objetiva a obtenção das informações através de passos progressivos, partindo do geral para o particular, num processo que integra o grupo pesquisado e o pesquisador. A metodologia utiliza a leitura da paisagem, que consiste em obter informações sobre as diversas formas de utilização do meio ambiente, realizada através de percursos sistemáticos de campo que permitam verificar as heterogeneidades do ambiente. Nessa leitura, cabem também reflexões sobre as causas das heterogeneidades observadas e a busca por justificativas através de entrevistas históricas. As entrevistas históricas subsidiam as informações obtidas através da leitura de paisagem e devem ser realizadas com informantes locais que possam fornecer mais elementos para explicar os fenômenos observados. Essas entrevistas devem ser realizadas através de participações nas atividades rotineiras, promovendo, como resultado final, um levantamento sobre a situação socioeconômica do grupo pesquisado, fatos marcantes, principais atividades desenvolvidas e transformações nas práticas de relação com os recursos naturais.

A representação de como o conhecimento sobre solos é percebido pelas populações locais pode ser através da elaboração de mapas das áreas de cultivo e/ou uso não agrícola, destacando as diferenças de paisagem identificadas, tipos de terra e vegetação. Essas estratégias podem constituir ferramentas definidoras para a melhor representação das relações entre agricultores, percepção espacial e categorização dos solos (BARRERA-BASSOLS et al., 2006).

A participação no cotidiano das pessoas é considerada uma forma de conquistar a confiança dos agricultores e de obter uma melhor percepção da forma como vivem, de seus problemas sociais, de suas manifestações culturais, crenças e atividades cotidianas, além de facilitar a troca de informações, por gerar um ambiente de confiança através do convívio (OUDWATER \& MARTIN, 2003; CORREIA, 2005).

Caminhadas transversais pelas áreas de atuação do grupo em estudo podem revelar aspectos de variação de relevo, vegetação e algumas propriedades relacionadas aos solos como cor, textura e espessura, que são obtidas diretamente pelo diálogo com os agricultores (OUDWATER \& MARTIN, 2003). O envolvimento com o grupo em estudo e reconhecimento das relações sociais, econômicas e culturais fornecem subsídios para obtenção de informações mais específicas do conhecimento local sobre solos. Dessa forma, o pesquisador é capaz não só de entender as relações com base na sua experiência, mas levando em consideração também a forma de visão local, permitindo a interpretação 
e conceitualização de forma mais significativa (ARÉVALO \& LJUNG, 2006). Agregando essas informações, MATOS (2008), em estudo com quilombolas, em Minas Gerais, identificou quatro macroambientes com base, principalmente, no relevo e teor de umidade do solo, sendo estes: "carrasco", "encosta" ou "cultura vermelha", "vazante" e "brejo". Cada macroambiente, em uma visão mais aprofundada, foi subdivido com base na participação de outras propriedades como cor do solo, localização, estrutura, textura, vegetação e inter-relação dessas propriedades com o clima.

Avaliações etnopedológicas podem relacionar-se a aspectos do uso não agrícola do solo, como o trabalho desenvolvido por ALVES et al. (2005) em uma localidade rural na Paraíba, onde testaram a hipótese de que haveria um conjunto de conhecimentos subjacentes às decisões da população quanto ao uso dos solos para o trabalho artesanal de cerâmica. Foram descritos perfis por uma equipe técnica nas proximidades dos barreiros (locais onde a população local extraía "barro de loiça") e posteriormente os ceramistas-agricultores indicaram e nomearam os principais tipos de componentes do solo nesses mesmos locais. Após isso, ambos os conjuntos de amostras foram analisados para comparação. Quatro perfis foram classificados como Planossolo Nátrico e um como Planossolo Háplico (EMBRAPA, 2006). Os ceramistas-agricultores identificaram os materiais "barro de loiça", "terra", "piçarro", "cabeça do barro" e "pedra mole". A visão, o tato e o paladar foram usados para distinguir os materiais do solo. A ocorrência de fendas nos materiais, devido à presença de argilominerais expansivos, foi um critério identificado pelos ceramistas-agricultores para averiguar se o "barro de loiça" era adequado para a atividade artesanal.

Ainda na região nordeste, o trabalho desenvolvido por QUEIROZ \& NORTON (1992) no Vale do Acaraú, Ceará, com acompanhamento detalhado de um grupo de agricultores por um ano, permitiu o reconhecimento de cinco denominações usadas pelos agricultores para caracterizar diferentes materiais do solo: "coroa" ou "baixio", "barro vermelho", "arisco", "barro de louça" e "massapê". Essas denominações apresentaram correspondência com as classes de solo identificadas através do sistema de classificação de solos Brasileiro (EMBRAPA, 2006).

A avaliação da distribuição dos tipos de terra identificados e a comparação com o mapa pedológico foi a abordagem usada por CORREIA et al. (2007), no trabalho desenvolvido no norte de Minas Gerais, onde elaboraram um levantamento pedológico numa área sob Cerrado e identificaram que as principais classes de solos foram os Latossolos, Argissolos, Cambissolos e Neossolos Flúvicos. Através do uso de metodologias como visitas às casas dos agricultores, vivência na área de estudo, caminhadas transversais e elaboração de mapas participativos, foi possível identificar seis ambientes: "baixa", "alta", "tabuleiro", "pirambeira", "carrasco" e "chapada". Cada ambiente foi distribuído espacialmente e, organizando as informações do conhecimento local, construiu-se o mapa de terras. Comparando-se os mapas, constatou-se que o conhecimento formal e local compartilham a forma de organização do conhecimento em níveis hierárquicos, ainda que sob a última forma de conhecimento esse arranjo aconteça de forma intuitiva. Os autores ressaltam que adaptações são necessárias para uma comparação mais adequada entre o sistema local e técnico, pois este último usa aspectos que colocam áreas consideradas diferentes pelos agricultores em um mesmo nível, e sugerem que parâmetros adicionais sejam incorporados ao sistema de classificação técnico para contemplar essa especificidade.

A distinção dos ambientes pelos agricultores, com base em aspectos quantitativos de produção, é uma das características pesquisadas em zoneamentos locais, baseado principalmente em características do solo. ARAÚJO (2000), realizando estudo em um assentamento no Acre, percebeu que os agricultores baseavam-se na textura do solo para distribuir os cultivos na área.

Estudos com comunidades indígenas também são representativos na área etnopedológica (WINKLERPRINS \& BARRERA-BASSOLS, 2004; REYES-GARCIA et al., 2007), abordando aspectos linguísticos, caracterização de atributos das terras e vocações para uso agrícola (VALE JÚNIOR et al., 2007; ARAÚJO et al., 2009; MELO et al., 2010).

$\mathrm{O}$ uso de Sistemas de Informações Geográficas (SIG) na aquisição e sistematização das informações tem mostrado resultados satisfatórios por disponibilizar espacialmente as observações feitas pelos agricultores e possibilitar ainda uma comparação simplificada com a abordagem técnica. BARRERA-BASSOLS et al. (2006) usaram o SIG para representar a distribuição de classes de solos identificados pelos agricultores em uma localidade no México Central e compararam com um mapa de solos gerado pela classificação técnica, chegando a uma correlação espacial de $75 \%$ entre classes de solos identificadas sob as duas formas de conhecimento.

Ainda utilizando o SIG como uma ferramenta para melhor avaliação das informações obtidas, SICAT et al. (2005) consideraram que o 
conhecimento local requer uma modelagem para a classificação das terras que possibilite a avaliação de dados vagos ou nebulosos, e propuseram a utilização da "teoria dos conjuntos fuzzy" para modelagem dessas informações. Dessa forma, as combinações binárias "e" e "ou" para as propriedades cor, textura e profundidade foram as que se ajustaram melhor às regras do conhecimento local, gerando uma distribuição mais ajustada à realidade da informação.

Atividades que integrem de forma sustentável as relações homem e ambiente têm se tornado imprescindíveis sob o ponto de vista legal, concretizadas através da legislação ambiental. Dessa forma, a resolução nº 387/2006 do Conselho Nacional de Meio Ambiente - CONAMA (BRASIL, 2006) regula os procedimentos para o licenciamento ambiental de Projetos de Assentamento de Reforma Agrária. FERNANDES et al. (2008), aliando essas exigências a uma pesquisa etnopedológica, realizaram a avaliação de um acampamento com a finalidade de subsidiar os procedimentos para efetivação do projeto de assentamento. Com esse trabalho, foi possível identificar diferentes ambientes, os quais foram distribuídos para uso de acordo com o Sistema de Capacidade de Uso das Terras (LEPSCH, 1983), proporcionando dessa forma uma utilização mais adequada das terras e a efetivação de uma melhoria na qualidade de vida das pessoas envolvidas por proporcionar uma adequada distribuição de terras.

FREITAS (2009), realizando trabalho em assentamento rural em Minas Gerais, propôs a etnopedologia como ferramenta mediadora no diálogo sobre o planejamento socioespacial desse ambiente. Usando metodologias participativas, foi possível identificar fatores ambientais e relacionálos com limitações e possibilidades de uso, as quais, combinadas com a forma dominante de organização social do grupo, foram consideradas, na elaboração do Plano de Desenvolvimento do Assentamento (PDA), documento que define as bases para o seu estabelecimento.

Percebe-se que pesquisas com propósitos integradores podem fornecer elementos importantes para os grupos sociais avaliados, como a possibilidade de obtenção de estruturas legalizadas para a sua produção, como é o estabelecimento de assentamentos rurais, bem como para a comunidade científica, como forma de ampliação dos elementos de estudo, pesquisa e divulgação dos trabalhos de forma mais acessível para os trabalhadores do campo e tomadores de decisão.

Para que os estudos etnopedológicos possam continuar ganhando representatividade,
SILLITOE \& MARZANO (2009) discutem que é necessário que os estudos continuem buscando engajar agências de fomento e garantindo mais espaço para pesquisa. No entanto, avaliam que devem ocorrer mudanças dos discursos dominantes de desenvolvimento para permitir que abordagens mais ousadas e com visões atualmente consideradas inviáveis possam ser desenvolvidas.

\section{CONCLUSÃO}

As etnociências preveem avaliações envolvendo pesquisadores e representantes do conhecimento local de forma a compartilhar as diferentes formas do conhecimento e a etnopedologia, como uma vertente das etnociências, busca estudar as relações e conhecimentos sobre solos de uma região, geradas por determinados grupos de pessoas e tem demonstrado sua importância com desempenho crescente de publicações e contribuído na divulgação sobre solos de forma direta fora do meio científico. Vale destacar que o solo e o ser humano são os elementos integradores da etnopedologia, no entanto, as relações com os demais recursos naturais não podem e nem devem ser desconsideradas. A etnopedologia destaca-se como uma importante ferramenta na busca por uma abordagem mais integradora da ciência do solo, proporcionando uma visão científica mais engajada com as populações humanas consideradas tradicionais ou locais.

\section{REFERÊNCIAS}

AGRAWAL, A. Indigenous and scientific knowledge. Indigenous Knowledge and Development Monitor, v.3, n.3, p.3-38, 1995.

ALVES, A.G.C. Do "barro de loiça" a "loiça de barro": caracterização etnopedológica de um artesanato camponês no agreste paraibano. 2004. 179f. Tese (Programa de Pós-graduação em Ecologia e Recursos naturais) - Universidade Federal de São Carlos, SP.

ALVES, A.G.C.; MARQUES, J.G.W. Etnopedologia: uma nova disciplina? In: VIDAL-TORRADO, P. et al. Tópicos em ciência do solo. Viçosa: Sociedade Brasileira de Ciência do Solo, 2005. V.4, cap.8, p.321-344.

ALVES, A.G.C. et al. Caracterização etnopedológica de planossolos utilizados em cerâmica artesanal no agreste paraibano. Revista Brasileira de Ciência do Solo, v.29, p.379388, 2005. Disponível em: <http://dx.doi.org/10.1590/S010006832005000300008>. Acesso em: 02 jan. 2012. doi: 10.1590/ S0100-06832005000300008.

ARAÚJO, E.A. Caracterização de solos e modificações provocadas pelo uso agrícola no assentamento Favo de Mel, na região do Purus-Acre. 2000. 122f. Dissertação (Mestrado em Solos e Nutrição de Plantas) - Universidade Federal de Viçosa, MG. 
ARAÚJO, J.L. et al. Atributos do solo e distinção de pedoambientes para a agricultura na terra indígena Mbya em Ubatuba (SP). Revista Brasileira de Ciências do Solo, v.33, n.6, p.1775-1776, 2009. Disponível em: <http://www.scielo.br/scielo. php?script=sci_arttext\&pid $=$ S0100-06832009000600025\&lng $=\mathrm{e}$ $\mathrm{n} \& \mathrm{nrm}=\mathrm{iso} \& \mathrm{t} \operatorname{lng}=\mathrm{pt}>$. Acesso em: 08 maio, 2012. doi: 10.1590/ S0100-06832009000600025.

ARÉVALO, K.M.; LJUNG, M. Action research on land management in the Western Amazon, Peru - A research process, its outcomes and the research's role. Systemic Practice and Action Research, v.19, n.4, p.309-324, 2006. Disponível em: <http:// www.springerlink.com/content/j3x1183383264071>. Acesso em: 08 maio, 2012. doi: 10.1007/s11213-006-9020-x.

BARRERA-BASSOLS, N.; ZINCK, J.A. Ethnopedology in a worldwide perspective: an annotated bibliography. The Netherlands: ITC Publication, 2000. 632p.

BARRERA-BASSOLS, N.; ZINCK, J.A. Ethnopedology: a worldwide view on the soil knowledge of local people. Geoderma, v.111, p.171-195, 2003. Disponível em: <http://www. sciencedirect.com/science/article/pii/S001670610200263X>. Acesso em: 02 jan. 2012. doi: 10.1016/S0016-7061(02)00263-X.

BARRERA-BASSOLS, N. et al. Local soil classification and comparison of indigenous and technical soil maps in a Mesoamerican community using spatial analysis. Geoderma, v.135, p.140-162, 2006. Disponível em: <http://www.sciencedirect. com/science/article/pii/S0016706105003083>. Acesso em: 02 jan. 2012. doi:10.1016/j.geoderma.2005.11.010.

BARRIOS, E.; TREJO, M.T. Implications of local soil knowledge for integrated soil management in Latin America. Geoderma, v.111, p.217-231, 2003. Disponível em: <http://www.sciencedirect. com/science/article/pii/S0016706102002653>. Acesso em: 02 jan. 2012. doi:10.1016/S0016-7061(02)00265-3.

BIRMINGHAM, D.M. Local knowledge of soils: the case of contrast in Côte d'Ivoire. Geoderma, v.111, p.481-502, 2003. Disponível em: <http://www.sciencedirect.com/science/article/pii/ S0016706102002781>. Acesso em: 02 jan. 2012. doi:10.1016/ S0016-7061(02)00278-1.

BRASIL. Resolução n.292 de 08 de julho de 1992. Regulamentação complementar da resolução CSN n.196/96. Diretrizes e normas regulamentadoras de pesquisas envolvendo seres humanos. Diário Oficial da República Federativa do Brasil, Poder Executivo, Brasília, DF, 15 set. 1999, Seção I, p.33.

BRASIL. Resolução n.387 de 27 de Dezembro de 2006. Estabelece procedimentos para o licenciamento ambiental de projetos de assentamentos de reforma agrária e dá outras providências. Diário Oficial da República Federativa do Brasil, Poder Executivo, Brasília, DF, 29 dez. 2006, Seção I, p.665-668.

BRIDGES, E.M. et al. Soils and education: the role of ISRIC. Wageningen: ISRIC - International Soil Reference and Information Centre, 1996. 12p. (Work paper and preprint).

CORREIA, J.R. Pedologia e conhecimento local: proposta metodológica de interlocução entre saberes construídos por pedólogos e agricultores em área de Cerrado em Rio Pardo de Minas, MG. 2005. 234f. Tese (Doutorado em Agronomia - Área de concentração Ciência do Solo) - Universidade Federal Rural do Rio de Janeiro, Seropédica, RJ.
CORREIA, J.R. et al. Relações entre o conhecimento de agricultores e de pedólogos sobre solos: estudo de caso em Rio Pardo de Minas, MG. Revista Brasileira de Ciência do Solo, v.31, p.1045-1057, 2007. Disponível em: <http://dx.doi.org/10.1590/ S0100-06832007000500021>. Acesso em: 02 jan. 2012. doi: 10.1590/S0100-06832007000500021.

DUFUMIER, M. Projetos de desenvolvimento agrícola. Manual para especialistas. Tradução de Vitor de Athayde Couto. Salvador: EDUFBA, 2007. 328p.

EMBRAPA (EMPRESA BRASILEIRA DE PESQUISA AGROPECUÁRIA). Centro Nacional de Pesquisa de Solos. Sistema brasileiro de classificação de solos. 2.ed. Rio de Janeiro, 2006. 306p

FAO (FOOD AND AGRICULTURE ORGANIZATION OF THE UNITED NATIONS). Building on gender, agrodiversity and local knowledge. Roma, 2004. 177p. (FAO Publication).

FERNANDES, L.A. et al. Relação entre o conhecimento local, atributos químicos e físicos do solo e uso das terras. Revista Brasileira de Ciência do Solo, v.32, p.1355-1365, 2008. Disponível em: <http://www.scielo.br/scielo.php?script=sci_ arttext $\&$ pid $=$ S0100 $-06832008000300043 \& \operatorname{lng}=$ en $\& n r m=i s o$ \&tlng=pt $>$. Acesso em: 02 jan. 2012. doi: 10.1590/S010006832008000300043

FREITAS, H.R. Contribuição da etnopedologia no planejamento da ocupação e uso do solo em assentamentos rurais. 2009. 174f. Tese (Doutorado em Solos e Nutrição de Plantas) - Universidade Federal de Viçosa, MG.

HARTEMINK, A.E.; MCBRATNEY,A. A soil science renaissance. Geoderma, v.148, p.123-129, 2008. Disponível em: <http://www. sciencedirect.com/science/article/pii/S0016706108002802>. Acesso em: 24 jan. 2012. doi:10.1016/j.geoderma.2008.10.006

LEPSCH, I.P. Levantamento utilitário do meio físico e classificação de terras no sistema de capacidade de uso: $4^{\text {a }}$ aproximação. Campinas: Sociedade Brasileira de Ciência do Solo, 1983. $175 \mathrm{p}$.

MARTIN, G.J. Ethnobotany: a methods manual. London: Chapman \& Hall, 1995. 292p.

MATOS, L.V. Conhecimentos na análise de ambientes: a pedologia e o saber local em comunidade quilombola do norte de Minas Gerais. 2008. 167f. Dissertação (Mestrado em solos e nutrição de plantas) - Universidade Federal de Viçosa, MG.

MEGONIGAL, J.P. et al. "Dig it": How an exhibit breathed life into soils education. Soil Science Society of American Journal, v.74, p.706-716, 2010. Disponível em: <http://hdl. handle.net/10088/11247>. Acesso em: 24 jan. 2012. doi:10.2136/ sssaj2009.0409.

MELO, V.F. et al. Solos da área indígena Yanomami no médio rio Catrimani, Roraima. Revista Brasileira de Ciências do Solo, v.34, p.487-496, 2010. Disponível em: <http://www.scielo.br/scielo. php?script=sci_arttext\&pid=S0100-06832010000200022\&lng=en $\& \mathrm{nrm}=\mathrm{iso} \& \operatorname{tlng}=\mathrm{pt}>$. Acesso em: 08 maio, 2012. doi: 10.1590/ S0100-06832010000200022.

MUGGLER, C.C et al. Educação em solos: princípios, teoria e métodos. Revista Brasileira de Ciência do Solo, v.30, p.733- 
740, 2006. Disponível em: <http://www.scielo.br/scielo.php ?script $=$ sci_arttext $\&$ pid $=$ S0100-06832006000400014\&lng $=$ e n\&nrm=iso>. Acesso em: 24 jan. 2012. doi: 10.1590/S010006832006000400014

NIEMEIJER, D.; MAZZUCATO, V. Moving beyond indigenous soil taxonomies: local theories of soils for sustainable development. Geoderma, v.111, p.403-424, 2003. Disponível em: <http://www. sciencedirect.com/science/article/pii/S0016706102002744>. Acesso em: 02 jan. 2012. doi:10.1016/S0016-7061(02)00274-4.

OUDWATER, N.; MARTIN, A. Methods and issues in exploring local knowledge of soils. Geoderma, v.111, p.387-401, 2003. Disponível em: <http://www.sciencedirect.com/science/article/pii/ S0016706102002732>. Acesso em: 02 jan. 2012. doi:10.1016/ S0016-7061(02)00273-2.

PAYTON, R.W. et al. Contrasting approaches to integrating indigenous knowledge about soils and scientific soil survey in East Africa and Bangladesh. Geoderma, v.111, p.355-386, 2003. Disponível em: <http://www.sciencedirect.com/science/article/pii/ S0016706102002720>. Acesso em: 02 jan. 2012. doi:10.1016/ S0016-7061(02)00272-0.

QUEIROZ, J.S.; NORTON, B.E. An assessment of an indigenous soil classification used in the caatinga region of Ceará State, Northeast Brazil. Agricultural Systems, v.39, p.289-305, 1992. Disponível em: <http://www.sciencedirect.com/science/ article/pii/0308521X9290101S >. Acesso em: 02 jan. 2012. doi:10.1016/0308-521X(92)90101-S.

REYEZ-GARCÍA, V. et al. Economic development and local ecological knowledge: a deadlock? Quantitative research from a native Amazonian society. Human Ecology, v.35, p.371377, 2007. Disponível em: <http://www.springerlink.com/ content/6433875148870242>. Acesso em: 08 maio, 2012. doi: $10.1007 / \mathrm{s} 10745-006-9069-2$.

SICAT, R.S. et al. Fuzzy modelling of farmers' knowledge for land suitability classification. Agricultural systems, v.83, p.4975, 2005. Disponível em: <http://www.sciencedirect.com/science/
article/pii/S0308521X0400054X>. Acesso em: 23 jan. 2012. doi:10.1016/ j.agsy. 2004.03.002.

SILLITOE, P; MARZANO, M. Future of indigenous knowledge research in development. Futures, v.41, p.13-23, 2009. Disponível em: <http://www.sciencedirect.com/science/article/pii/ S0016328708001109>. Acesso em: 23 jan. 2012. doi: 10.1016/j. futures.2008.07.004.

TOLEDO, V.M. Indigenous knowledge of soils: an ethnoecological conceptualization. In: BARRERA-BASSOLS, N.; ZINCK, J.A. Ethnopedology in a worldwide perspective. An annotated bibliography. The Netherlands: ITC Publication, 2000. 632p.

VALE JÚNIOR, J.F. et al. Etnopedologia e transferência de conhecimento: diálogos entre os saberes indígena e técnico na terra indígena Malacheta, Roraima. Revista Brasileira de Ciências do Solo, v.31, p.403-412, 2007. Disponível em: <http://www.scielo.br/scielo.php?script=sci_arttext\&pid=S0100$06832007000200023 \& \operatorname{lng}=\mathrm{en} \& \mathrm{nrm}=\mathrm{iso} \& \mathrm{t} \operatorname{lng}=\mathrm{pt}>$. Acesso em: 08 maio, 2012. doi: 10.1590/S0100-06832007000200023.

WILDING, L.P.; LIN, H. Advancing the frontiers of soil science towards a geosciences. Geoderma, v.131 p.257-274, 2006. Disponível em: <http://www.sciencedirect.com/science/article/pii/ S0016706105000790>. Acesso em: 23 jan. 2012. doi:10.1016/j. geoderma.2005.03.028.

WILLIAMS, B.J.; ORTIZ-SOLORIO, C.A. Middle American folk soil taxonomy. Annals of the Association of American Geographers, v.71, n.3, p.335-358, 1981. Disponível em: <http://onlinelibrary.wiley.com/doi/10.1111/j.1467-8306.1981. tb01361.x/abstract?GlobalMessage=0>. Acesso em: 03 jan. 2012. doi: 10.1111/j.1467-8306.1981.tb01361.x.

WINKLERPRINS, A.M.G.A.; BARRERA-BASSOLS, N. Latin American ethnopedology: a vision of its past, present and future. Agriculture and human values, v.21, p.139156, 2004. Disponível em: <http://www.springerlink.com/ content/r6753p7wr63413h7>. Acesso em: 08 maio, 2012. doi: 10.1023/B:AHUM.0000029405.37237.c8. 